\title{
DESCRIPTION OF POSETS CRITICAL WITH RESPECT TO THE NONNEGATIVITY OF THE QUADRATIC TITS FORM
}

\author{
V. M. Bondarenko and M. V. Stepochkina
}

UDC 512.64+512.56

\begin{abstract}
We present the complete description of finite posets whose Tits form is not nonnegative but all proper subsets of which have nonnegative Tits forms. A similar result for positive forms was obtained by the authors earlier.
\end{abstract}

\section{Introduction}

In [1], for a finite quiver (oriented graph) $Q$ with the set of vertices $Q_{0}$ and the set of arrows $Q_{1}$, Gabriel introduced a quadratic form $q_{Q}: \mathbb{Z}^{Q_{0}} \rightarrow \mathbb{Z}$ and called it the quadratic Tits form of the quiver $Q$ :

$$
q_{Q}(z)=\sum_{i \in Q_{0}} z_{i}^{2}-\sum_{i \rightarrow j} z_{i} z_{j}
$$

where $i \rightarrow j$ runs over the set $Q_{1}$. In [1], he also proved that the quiver has a finite representation type over the field $k$ (i.e., finitely many indecomposable representations, to within an isomorphism) if and only if its Tits form is positive. This Gabriel's work laid the foundations of a new field of algebra dealing with the investigation of the relationships between the properties of representations of various objects and the properties of quadratic forms associated with these objects.

Later, the quadratic Tits forms for quivers with relations and posets were studied by Brenner [2] and Drozd [3], respectively. In the general case of matrix problems without relations, the Tits form was introduced by Kleiner and Roiter [4].

In [3], it is shown that a poset has a finite representation type if and only if its Tits form is weakly positive, i.e., takes positive value on any nonzero vector with nonnegative coordinates (representations of posets were introduced by Nazarova and Roiter in [5]). Note that the Tits form of a quiver is weakly positive if and only if it is positive. For posets, this is not true. Therefore, the investigations of posets with positive Tits form seems to be quite natural (at least, from the formal point of view). Posets of this sort were studied in [6-14]. Moreover, in the cited works, the posets with positive Tits form were also studied from the viewpoint of their properties directly connected with the categories of representations.

The present paper is devoted to the investigation of posets with nonnegative Tits form and continues our investigation of posets of this sort originated in [15].

\section{Formulation of the Main Result}

Let $S$ be a finite poset that does not contain element 0 . Its quadratic Tits form is defined as a quadratic form $q_{S}: \mathbb{Z}^{S \cup 0} \rightarrow \mathbb{Z}$ given by the equality

Institute of Mathematics, Ukrainian National Academy of Sciences, Kiev, Ukraine.

Translated from Ukrains'kyi Matematychnyi Zhurnal, Vol.61, No. 5, pp.611-624, May, 2009. Original article submitted December 23, 2008. 


$$
q_{S}(z)=z_{0}^{2}+\sum_{i \in S} z_{i}^{2}+\sum_{i<j, i, j \in S} z_{i} z_{j}-z_{0} \sum_{i \in S} z_{i}
$$

A poset $S$ is called critical with respect to positivity (nonnegativity) of the quadratic Tits form or, briefly, $P$-critical ( NP-critical) if the Tits form of any its proper subset is positive (negative) but the Tits form of the entire $S$ is not a form of the same type.

The width of a poset $S$ is defined as the maximum number of its pairwise incomparable elements. A poset dual to $S$ is denoted by $S^{\mathrm{op}}$. In other words, $S^{\mathrm{op}}=S$ as ordinary sets and, moreover $x<y$ in $S^{\mathrm{op}}$ if and only if $x>y$ in $S$. The posets $S$ and $T$ are called antiisomorphic if $S$ is isomorphic to $T^{\mathrm{op}}$.

The list of all $P$-critical posets can be found in [10]. This list is also presented in Table 1 (see the last section). The aim of the present paper is to describe all $N P$-critical sets .

The following theorem is true:

Theorem 1. The NP-critical posets are exhausted, to within isomorphisms and antiisomorphisms, by posets 1-115 presented in Table 2 (see Section 5).

We now make several comments on Table 2.

The poset with number $i$ in Table 2 is denoted by $N_{i}$. If the set $N_{i}$ has width 2 and, in Table 2, it is indicated that $i=j^{\prime}$, then this means that $N_{i}$ can be obtained from $N_{j}$ by replacing its unique maximum point with the unique minimum point. The same is true for the case $i=j^{\prime \prime}=\left(j^{\prime}\right)^{\prime}$ (it is necessary to compare $N_{i}$ with $N_{j^{\prime}}$ ). If the set $N_{i}$ has width 3 and, in Table 2, it is indicated that $i=j^{\prime}$, then this means that the procedure described above should be applied not to $N_{i}$ and $N_{j}$ but to their connected components (direct terms) of width 2. The same is true for the case $i=j^{\prime \prime}$.

Any posets $S$ and $T$ obtained from each other as a result of operations of this sort are called 0 -isomorphic. Excluding the sets with the numbers $i=j^{\prime}$ and $i=j^{\prime \prime}$ from Table 2, we obtain the description of $N P$-critical sets to within 0 -isomorphism and duality.

The same is true for Table 1 .

\section{Min-Equivalent Posets}

We now present several facts about min-equivalent posets. This type of equivalence was introduced by one of the authors (V.B.) in [7]. Its detailed investigation was carried out in [10].

Let $S$ be a poset. For the minimum element $a \in S$, by $S_{a}^{\uparrow}$ we denote a poset that coincides with $S$ as a set with the same relation of ordering on $S \backslash\{a\}$ but, in $S_{a}^{\uparrow}$, the element $a$ is already the maximum element and, in addition, $a$ is comparable with $x$ in $T$ if and only if $a$ is incomparable with $x$ in $S$. We write $S_{x y}^{\uparrow \uparrow}$ instead of $\left(S_{x}^{\uparrow}\right)_{y}^{\uparrow}, \quad S_{x y z}^{\uparrow \uparrow}$ instead of $\left(\left(S_{x}^{\uparrow}\right)_{y}^{\uparrow}\right)_{z}^{\uparrow}$, etc.

A poset $T$ is called min-equivalent to a poset $S$ if $T$ is equal to a certain poset of the form

$$
\bar{S}=S_{x_{1} x_{2} \ldots x_{p}}^{\uparrow \uparrow}, \quad p \geq 0
$$

where $x_{i}$ is the minimum element of $S_{x_{1} x_{2} \ldots x_{i-1}}^{\uparrow \uparrow}$ for any $i \in\{1, \ldots, p\}$. For $p=0$, we assume that $\bar{S}=S$. It is not required that the elements $x_{1}, x_{2}, \ldots, x_{p}$ must be different.

The min-equivalence of posets is denoted by $\cong_{\min }$.

Note that the notion of min-equivalence can be naturally extended to the notion of min-isomorphism if we assume that the posets $S$ and $S^{\prime}$ are min-isomorphic if there exists a poset $T$ min-equivalent to $S$ and isomorphic to $S^{\prime}$. 
Let $S$ be a poset. A finite sequence $\alpha=\left(x_{1}, x_{2}, \ldots, x_{p}\right)$ of elements $x_{i} \in S$ is called min-admissible if the expression $\bar{S}=S_{x_{1} x_{2} \ldots x_{p}}^{\uparrow \uparrow \uparrow}$ is meaningful (the case $p=0$ is not excluded). In this case, we also write $\bar{S}=S_{\alpha}^{\uparrow}$.

The set of all min-admissible sequences is denoted by $\mathcal{P}(S)$ and the set of all sequences of this sort without repetitions by $\mathcal{P}_{1}(S)$. Let $[\alpha]_{S}$ be a subset in $S$ formed by all elements $x_{i}$ of the sequence $\alpha \in \mathcal{P}_{1}(S)$. Note that, for the min-equivalent posets $S$ and $T$, a sequence $\alpha \in \mathcal{P}_{1}(S)$ such that $T=S_{\alpha}^{\uparrow}$ does not always exist (see [10], Sec. 6).

A subset $X$ is called lower if $x \in X$ whenever $x<y$ and $y \in X$. The notation $X<Y$ for the subsets $S$ means that $x<y$ for any $x \in X$ and $y \in Y$. Note that $Z<\varnothing$ and $\varnothing<Z$ for any subset $Z$.

According to Corollaries 5 and 9 from [10], the following two assertions are true:

(1) in $\mathcal{P}_{1}(S)$, there exists a sequence $\alpha$ such that $[\alpha]_{S}=X$ if and only if $X$ is a lower subset;

(2) if $\alpha, \beta \in \mathcal{P}_{1}(S)$ and $[\alpha]_{S}=[\beta]_{S}$, then $S_{\alpha}^{\uparrow}=S_{\beta}^{\uparrow}$.

Hence, for the lower subset $X$, one can naturally define the poset $S_{X}^{\uparrow}$ by setting $S_{X}^{\uparrow}=S_{\alpha}^{\uparrow}$, where $\alpha \in \mathcal{P}_{1}(S)$ is an arbitrary sequence such that $[\alpha]_{S}=X$. It is easy to see (Proposition 6 in [10]) that $a<b$ in $\bar{S}=S_{X}^{\uparrow}$ if and only if one of the following conditions is satisfied:

(a) $a<b$ in $S$ and either $a, b \in X$ or $a, b \notin X$;

(b) $a \gtrless b$ in $S$ and $b \in X, a \notin X$.

This, in particular, implies that if $Z$ is a lower subset in $X$ such that $Z<S \backslash X$, then $Z$ is a lower subset also in $S_{X}^{\uparrow}$.

An algorithm for the description of all posets min-equivalent to a fixed poset $S$ is proposed (and justified) in [10]. This algorithm consists of the following steps:

I. To describe all lower subsets $X \neq S$ in $S$ and construct the poset $S_{X}^{\uparrow}$ for each of these sets.

II. To describe all couples $(Y, X)$ formed by a proper lower subset $Y$ in $S$ and a nonempty lower subset $X$ in $Y$ such that $X<S \backslash Y$ and construct, for each couple of this type, the poset $S_{Y X}^{\uparrow \uparrow}=\left(S_{Y}^{\uparrow}\right)_{X}^{\uparrow}$.

III. To select a single poset from each class of isomorphic sets in the families of posets constructed in Steps I and II.

Subsets $X$ and $X^{\prime}$ indicated in Step I are called strongly isomorphic if there exists an automorphism $\varphi: S \rightarrow$ $S$ such that $\varphi(X)=X^{\prime}$ (as subposets). Similarly, two couples $(Y, X)$ and $\left(Y^{\prime}, X^{\prime}\right)$ indicated in Step II are called strongly isomorphic if there exists an automorphism $\varphi: S \rightarrow S$ such that $\varphi(Y)=Y^{\prime}$ and $\varphi(X)=X^{\prime}$. Clearly, it suffices to describe the subsets in Step I and the couples of subsets in Step II to within a strong isomorphism.

\section{Proof of Theorem 1}

First, we formulate one of the two main results obtained in [15] and used in what follows.

It is known that the following posets (in [15], they are called the critical Nazarova posets) play an important role in the theory of representations:

$\mathcal{N}_{1}=\{1,2,3,4,5\}$, where all elements are pairwise incomparable: 
$\mathcal{N}_{2}=\{1,2,3,4,5 \mid 4 \prec 5\}:$

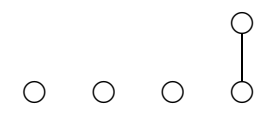

$\mathcal{N}_{3}=\{1,2,3,4,5,6,7 \mid 1 \prec 2,3 \prec 4,5 \prec 6 \prec 7\}:$<smiles>OOOOOO</smiles>

$\mathcal{N}_{4}=\{1,2,3,4,5,6,7,8 \mid 2 \prec 3 \prec 4,5 \prec 6 \prec 7 \prec 8\}:$

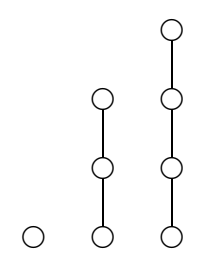

$\mathcal{N}_{5}=\{1,2,3,4,5,6,7,8,9 \mid 2 \prec 3,4 \prec 5 \prec 6 \prec 7 \prec 8 \prec 9\}:$

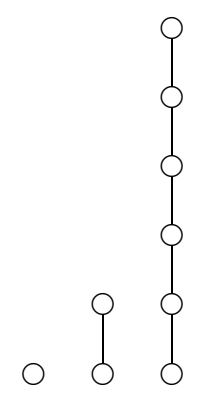

$\mathcal{N}_{6}=\{1,2,3,4,5,6,7,8,9 \mid 1 \prec 2 \prec 3 \prec 4 \prec 5,6 \prec 7,8 \prec 9,6 \prec 9\}:$

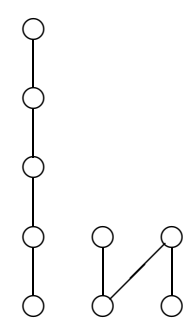

We can now formulate the required result from [15].

Theorem 2. A poset $S$ is NP-critical if and only if it is min-equivalent to a critical Nazarova poset. 
Thus, all NP-critical posets can be obtained as follows: It is necessary to take all critical Nazarova sets and describe all posets min-equivalent to each of these sets (in what follows, we always say "all" instead of "all to within an isomorphism"). However, if we use solely the definition of min-equivalence, then it is not completely clear how to realize the corresponding process in practice (because, from the formal point of view, it is infinite). To do this, we use the algorithm outlined in the previous section.

We now directly proceed to the proof of Theorem 1.

Step I. We describe (to within a strong isomorphism) all lower subsets in the critical Nazarova sets $\mathcal{N}_{1}-\mathcal{N}_{6}$ (see Sec. 4):

for $\mathcal{N}_{1} \quad A_{1,1}=\varnothing, A_{1,2}=\{1\}, A_{1,3}=\{1,2\}, A_{1,4}=\{1,2,3\}$, and $A_{1,5}=\{1,2,3,4\} ;$

for $\mathcal{N}_{2} A_{2,1}=\varnothing, A_{2,2}=\{1\}, A_{2,3}=\{4\}, A_{2,4}=\{1,2\}, A_{2,5}=\{1,4\}, A_{2,6}=\{4,5\}, A_{2,7}=\{1,2,3\}$, $A_{2,8}=\{1,2,4\}, A_{2,9}=\{1,4,5\}, A_{2,10}=\{1,2,3,4\}$, and $A_{2,11}=\{1,2,4,5\} ;$

for $\mathcal{N}_{3} A_{3,1}=\varnothing, A_{3,2}=\{1\}, A_{3,3}=\{5\}, A_{3,4}=\{1,2\}, A_{3,5}=\{1,3\}, A_{3,6}=\{1,5\}, A_{3,7}=\{5,6\}$, $A_{3,8}=\{1,2,3\}, A_{3,9}=\{1,2,5\}, A_{3,10}=\{1,3,5\}, A_{3,11}=\{1,5,6\}, A_{3,12}=\{5,6,7\}, A_{3,13}=$ $\{1,2,3,4\}, A_{3,14}=\{1,2,3,5\}, A_{3,15}=\{1,2,5,6\}, \quad A_{3,16}=\{1,3,5,6\}, \quad A_{3,17}=\{1,5,6,7\}$, $A_{3,18}=\{1,2,3,4,5\}, A_{3,19}=\{1,2,3,5,6\}, A_{3,20}=\{1,2,5,6,7\}, A_{3,21}=\{1,3,5,6,7\}, A_{3,22}=$ $\{1,2,3,4,5,6\}$, and $A_{3,23}=\{1,2,3,5,6,7\}$;

for $\mathcal{N}_{4} \quad A_{4,1}=\varnothing, A_{4,2}=\{1\}, A_{4,3}=\{2\}, A_{4,4}=\{5\}, A_{4,5}=\{1,2\}, A_{4,6}=\{1,5\}, \quad A_{4,7}=$ $\{2,3\}, A_{4,8}=\{2,5\}, A_{4,9}=\{5,6\}, A_{4,10}=\{1,2,3\}, A_{4,11}=\{1,2,5\}, \quad A_{4,12}=\{1,5,6\}$, $A_{4,13}=\{2,3,4\}, A_{4,14}=\{2,3,5\}, A_{4,15}=\{2,5,6\}, \quad A_{4,16}=\{5,6,7\}, \quad A_{4,17}=\{1,2,3,4\}$, $A_{4,18}=\{1,2,3,5\}, \quad A_{4,19}=\{1,2,5,6\}, \quad A_{4,20}=\{1,5,6,7\}, \quad A_{4,21}=\{2,3,4,5\}, \quad A_{4,22}=$ $\{2,3,5,6\}, A_{4,23}=\{2,5,6,7\}, A_{4,24}=\{5,6,7,8\}, A_{4,25}=\{1,2,3,4,5\}, A_{4,26}=\{1,2,3,5,6\}$, $A_{4,27}=\{1,2,5,6,7\}, \quad A_{4,28}=\{1,5,6,7,8\}, \quad A_{4,29}=\{2,3,4,5,6\}, \quad A_{4,30}=\{2,3,5,6$, $7\}, A_{4,31}=\{2,5,6,7, \quad 8\}, \quad A_{4,32}=\{1,2,3,4,5,6\}, \quad A_{4,33}=\{1,2,3,5,6,7\}, \quad A_{4,34}=$ $\{1,2,5,6,7,8\}, \quad A_{4,35}=\{2,3,4,5, \quad 6, \quad 7\}, \quad A_{4,36}=\{2,3,5,6,7,8\}, \quad A_{4,37}=\{1,2,3$, $4,5,6,7\}, A_{4,38}=\{1,2,3,5,6,7,8\}$, and $A_{4,39}=\{2,3,4,5,6,7,8\}$;

for $\mathcal{N}_{5} \quad A_{5,1}=\varnothing, A_{5,2}=\{1\}, A_{5,3}=\{2\}, A_{5,4}=\{4\}, A_{5,5}=\{1,2\}, A_{5,6}=\{1,4\}$, $A_{5,7}=\{2,3\}, \quad A_{5,8}=\{2,4\}, A_{5,9}=\{4,5\}, A_{5,10}=\{1,2,3\}, A_{5,11}=\{1,2,4\}, A_{5,12}=$ $\{1,4,5\}, A_{5,13}=\{2,3,4\}, A_{5,14}=\{2,4,5\}, A_{5,15}=\{4,5,6\}, A_{5,16}=\{1,2,3,4\}, A_{5,17}=$ $\{1,2,4,5\}, A_{5,18}=\{1,4,5,6\}, A_{5,19}=\{2,3,4,5\}, A_{5,20}=\{2,4,5,6\}, \quad A_{5,21}=\{4,5,6,7\}$, $A_{5,22}=\{1,2,3,4,5\}, \quad A_{5,23}=\{1,2,4,5,6\}, \quad A_{5,24}=\{1,4,5,6,7\}, \quad A_{5,25}=\{2,3,4,5,6\}$, $A_{5,26}=\{2,4,5,6,7\}, A_{5,27}=\{4,5,6,7,8\}, A_{5,28}=\{1,2,3,4,5,6\}, A_{5,29}=\{1,2,4,5,6,7\}$, $A_{5,30}=\{1,4,5,6,7,8\}, \quad A_{5,31}=\{2,3,4,5,6,7\}, \quad A_{5,32}=\{2,4,5,6,7,8\}, \quad A_{5,33}=\{4,5,6$, $7,8,9\}, \quad A_{5,34}=\{1,2,3,4,5,6,7\}, \quad A_{5,35}=\{1,2,4,5,6, \quad 7, \quad 8\}, \quad A_{5,36}=\{1,4,5,6,7,8$, $9\}, A_{5,37}=\{2,3,4,5,6,7,8\}, A_{5,38}=\{2,4,5,6,7,8,9\}, A_{5,39}=\{1,2,3,4,5,6,7,8\}, A_{5,40}=$ $\{1,2,4,5,6,7,8,9\}$, and $A_{5,41}=\{2,3,4,5,6,7,8,9\}$;

for $\mathcal{N}_{6} A_{6,1}=\varnothing, A_{6,2}=\{1\}, A_{6,3}=\{6\}, A_{6,4}=\{8\}, A_{6,5}=\{1,2\}, A_{6,6}=\{1,6\}, A_{6,7}=$ $\{1,8\}, A_{6,8}=\{6,7\}, A_{6,9}=\{6,8\}, A_{6,10}=\{1,2,3\}, A_{6,11}=\{1,2,6\}, A_{6,12}=\{1,2,8\}$, $A_{6,13}=\{1,6,7\}, A_{6,14}=\{1,6,8\}, A_{6,15}=\{6,7,8\}, A_{6,16}=\{6,8,9\}, \quad A_{6,17}=\{1,2,3,4\}$, $A_{6,18}=\{1,2,3,6\}, \quad A_{6,19}=\{1,2,3,8\}, \quad A_{6,20}=\{1,2,6,7\}, \quad A_{6,21}=\{1,2,6,8\}, \quad A_{6,22}=$ $\{1,6,7,8\}, A_{6,23}=\{1,6,8,9\}, A_{6,24}=\{6,7,8,9\}, A_{6,25}=\{1,2,3,4,5\}, A_{6,26}=\{1,2,3,4,6\}$, $A_{6,27}=\{1,2,3,4,8\}, \quad A_{6,28}=\{1,2,3,6,7\}, \quad A_{6,29}=\{1,2,3,6,8\}, \quad A_{6,30}=\{1,2,6,7$, $8\}, A_{6,31}=\{1,2,6,8,9\}, \quad A_{6,32}=\{1,6,7,8,9\}, \quad A_{6,33}=\{1,2,3,4,5,6\}, \quad A_{6,34}=$ 
$\{1,2,3,4,5,8\}, \quad A_{6,35}=\{1,2,3,4, \quad 6, \quad 7\}, \quad A_{6,36}=\{1,2,3,4,6,8\}, \quad A_{6,37}=\{1,2,3$, $6,7,8\}, A_{6,38}=\{1,2,3,6,8,9\}, A_{6,39}=\{1,2,6,7,8,9\}, A_{6,40}=\{1,2,3,4,5,6,7\}, A_{6,41}=$ $\{1,2,3,4,5,6,8\}, A_{6,42}=\{1,2,3,4,6,7,8\}, A_{6,43}=\{1,2,3,4,6,8,9\}, A_{6,44}=\{1,2,3,6,7,8,9\}$, $A_{6,45}=\{1,2,3,4,5,6,7,8\}, A_{6,46}=\{1,2,3,4,5,6,8,9\}$, and $A_{6,47}=\{1,2,3,4,6,7,8,9\}$.

By $N_{i, j}$ we denote the poset $S_{X}^{\uparrow}$ for $S=\mathcal{N}_{i}$ and $X=A_{i, j}$. Then it is easy to see that $\mathcal{N}_{1,1} \cong N_{115}$, $\mathcal{N}_{1,2} \cong N_{114}, \mathcal{N}_{1,3} \cong N_{45}, \mathcal{N}_{1,4} \cong N_{45}^{\mathrm{op}}, \mathcal{N}_{1,5} \cong N_{114}^{\mathrm{op}}, \mathcal{N}_{2,1} \cong N_{112}, \mathcal{N}_{2,2} \cong N_{43}, \mathcal{N}_{2,3} \cong N_{113}$, $\mathcal{N}_{2,4} \cong N_{1}^{\mathrm{op}}, \mathcal{N}_{2,5} \cong N_{44}, \mathcal{N}_{2,6} \cong N_{41}, \mathcal{N}_{2,7} \cong N_{41}^{\mathrm{op}}, \mathcal{N}_{2,8} \cong N_{44}^{\mathrm{op}}, \mathcal{N}_{2,9} \cong N_{1}, \mathcal{N}_{2,10} \cong N_{113}^{\mathrm{op}}, \mathcal{N}_{2,11} \cong$ $N_{43}^{\mathrm{op}}, \quad \mathcal{N}_{3,1} \cong N_{46}, \quad \mathcal{N}_{3,2} \cong N_{49}, \mathcal{N}_{3,3} \cong N_{50}, \mathcal{N}_{3,4} \cong N_{4}, \mathcal{N}_{3,5} \cong N_{53}, \mathcal{N}_{3,6} \cong N_{52}, \mathcal{N}_{3,7} \cong N_{47}$, $\mathcal{N}_{3,8} \cong N_{6}^{\mathrm{op}}, \mathcal{N}_{3,9} \cong N_{7}, \mathcal{N}_{3,10} \cong N_{54}^{\mathrm{op}}, \mathcal{N}_{3,11} \cong N_{51}, \mathcal{N}_{3,12} \cong N_{2}, \mathcal{N}_{3,13} \cong N_{2}^{\mathrm{op}}, \mathcal{N}_{3,14} \cong N_{51}^{\mathrm{op}}$, $\mathcal{N}_{3,15} \cong N_{7}^{\mathrm{op}}, \mathcal{N}_{3,16} \cong N_{54}, \mathcal{N}_{3,17} \cong N_{6}, \mathcal{N}_{3,18} \cong N_{47}^{\mathrm{op}}, \mathcal{N}_{3,19} \cong N_{52}^{\mathrm{op}}, \mathcal{N}_{3,20} \cong N_{4}^{\mathrm{op}}, \mathcal{N}_{3,21} \cong N_{53}^{\mathrm{op}}$, $\mathcal{N}_{3,22} \cong N_{50}^{\mathrm{op}}, \mathcal{N}_{3,23} \cong N_{49}^{\mathrm{op}}, \mathcal{N}_{4,1} \cong N_{55}, \mathcal{N}_{4,2} \cong N_{17}, \mathcal{N}_{4,3} \cong N_{62}, \mathcal{N}_{4,4} \cong N_{63}, \mathcal{N}_{4,5} \cong N_{15}^{\mathrm{op}}, \mathcal{N}_{4,6} \cong$ $N_{18}^{\mathrm{op}}, \mathcal{N}_{4,7} \cong N_{58}, \mathcal{N}_{4,8} \cong N_{69}, \mathcal{N}_{4,9} \cong N_{60}, \mathcal{N}_{4,10} \cong N_{13}^{\mathrm{op}}, \mathcal{N}_{4,11} \cong N_{66}^{\mathrm{op}}, \mathcal{N}_{4,12} \cong N_{16}^{\mathrm{op}}, \mathcal{N}_{4,13} \cong N_{11}$, $\mathcal{N}_{4,14} \cong N_{65}, \quad \mathcal{N}_{4,15} \cong N_{68}, \mathcal{N}_{4,16} \cong N_{56}, \mathcal{N}_{4,17} \cong N_{8}^{\mathrm{op}}, \mathcal{N}_{4,18} \cong N_{64}^{\mathrm{op}}, \mathcal{N}_{4,19} \cong N_{67}^{\mathrm{op}}, \mathcal{N}_{4,20} \cong N_{14}^{\mathrm{op}}$, $\mathcal{N}_{4,21} \cong N_{14}, \quad \mathcal{N}_{4,22} \cong N_{67}, \mathcal{N}_{4,23} \cong N_{64}, \mathcal{N}_{4,24} \cong N_{8}, \quad \mathcal{N}_{4,25} \cong N_{56}^{\text {op }}, \mathcal{N}_{4,26} \cong N_{68}^{\text {op }}, \mathcal{N}_{4,27} \cong N_{65}^{\text {op }}$, $\mathcal{N}_{4,28} \cong N_{11}^{\mathrm{op}}, \mathcal{N}_{4,29} \cong N_{16}, \mathcal{N}_{4,30} \cong N_{66}, \mathcal{N}_{4,31} \cong N_{13}, \mathcal{N}_{4,32} \cong N_{60}^{\mathrm{op}}, \mathcal{N}_{4,33} \cong N_{69}^{\mathrm{op}}, \mathcal{N}_{4,34} \cong N_{58}^{\mathrm{op}}$, $\mathcal{N}_{4,35} \cong N_{18}, \mathcal{N}_{4,36} \cong N_{15}, \mathcal{N}_{4,37} \cong N_{63}^{\mathrm{op}}, \mathcal{N}_{4,38} \cong N_{62}^{\mathrm{op}}, \mathcal{N}_{4,39} \cong N_{17}^{\mathrm{op}}, \mathcal{N}_{5,1} \cong N_{70}, \mathcal{N}_{5,2} \cong N_{27}$, $\mathcal{N}_{5,3} \cong N_{74}, \mathcal{N}_{5,4} \cong N_{84}^{\mathrm{op}}, \mathcal{N}_{5,5} \cong N_{25}^{\mathrm{op}}, \mathcal{N}_{5,6} \cong N_{29}, \mathcal{N}_{5,7} \cong N_{23}, \mathcal{N}_{5,8} \cong N_{95}, \mathcal{N}_{5,9} \cong N_{82}, \mathcal{N}_{5,10} \cong N_{19}^{\mathrm{op}}$, $\mathcal{N}_{5,11} \cong N_{94}, \quad \mathcal{N}_{5,12} \cong N_{31}, \mathcal{N}_{5,13} \cong N_{26}, \mathcal{N}_{5,14} \cong N_{96}, \mathcal{N}_{5,15} \cong N_{80}, \mathcal{N}_{5,16} \cong N_{71}^{\mathrm{op}}, \mathcal{N}_{5,17} \cong N_{101}^{\mathrm{op}}$, $\mathcal{N}_{5,18} \cong N_{30}^{\mathrm{op}}, \mathcal{N}_{5,19} \cong N_{23}, \mathcal{N}_{5,20} \cong N_{99}, \mathcal{N}_{5,21} \cong N_{77}, \mathcal{N}_{5,22} \cong N_{77}^{\mathrm{op}}, \mathcal{N}_{5,23} \cong N_{99}^{\mathrm{op}}, \mathcal{N}_{5,24} \cong N_{28}^{\mathrm{op}}$, $\mathcal{N}_{5,25} \cong N_{30}, \mathcal{N}_{5,26} \cong N_{101}, \mathcal{N}_{5,27} \cong N_{71}, \mathcal{N}_{5,28} \cong N_{80}^{\mathrm{op}}, \mathcal{N}_{5,29} \cong N_{96}^{\mathrm{op}}, \mathcal{N}_{5,30} \cong N_{26}^{\mathrm{op}}, \mathcal{N}_{5,31} \cong N_{31}^{\mathrm{op}}$, $\mathcal{N}_{5,32} \cong N_{94}^{\mathrm{op}}, \mathcal{N}_{5,33} \cong N_{19}, \mathcal{N}_{5,34} \cong N_{82}^{\mathrm{op}}, \mathcal{N}_{5,35} \cong N_{95}^{\mathrm{op}}, \mathcal{N}_{5,36} \cong N_{23}^{\mathrm{op}}, \mathcal{N}_{5,37} \cong N_{29}^{\mathrm{op}}, \mathcal{N}_{5,38} \cong N_{25}$, $\mathcal{N}_{5,39} \cong N_{84}^{\mathrm{op}}, \quad \mathcal{N}_{5,40} \cong N_{74}^{\mathrm{op}}, \mathcal{N}_{5,41} \cong N_{27}^{\mathrm{op}}, \mathcal{N}_{6,1} \cong N_{85}, \mathcal{N}_{6,2} \cong N_{93}, \mathcal{N}_{6,3} \cong N_{76}, \mathcal{N}_{6,4} \cong N_{97}^{\mathrm{op}}$, $\mathcal{N}_{6,5} \cong N_{91}, \mathcal{N}_{6,6} \cong N_{98}, \quad \mathcal{N}_{6,7} \cong N_{111}, \mathcal{N}_{6,8} \cong N_{37}, \mathcal{N}_{6,9} \cong N_{75}^{\mathrm{op}}, \mathcal{N}_{6,10} \cong N_{89}, \mathcal{N}_{6,11} \cong N_{100}$, $\mathcal{N}_{6,12} \cong N_{110}, \mathcal{N}_{6,13} \cong N_{39}, \mathcal{N}_{6,14} \cong N_{106}^{\text {op }}, \mathcal{N}_{6,15} \cong N_{104}^{\text {op }}, \mathcal{N}_{6,16} \cong N_{36}^{\text {op }}, \mathcal{N}_{6,17} \cong N_{86}, \mathcal{N}_{6,18} \cong N_{102}$, $\mathcal{N}_{6,19} \cong N_{109}, \mathcal{N}_{6,20} \cong N_{40}, \mathcal{N}_{6,21} \cong N_{107}^{\mathrm{op}}, \mathcal{N}_{6,22} \cong N_{103}^{\mathrm{op}}, \mathcal{N}_{6,23} \cong N_{108}^{\mathrm{op}}, \mathcal{N}_{6,24} \cong N_{32}^{\mathrm{op}}, \mathcal{N}_{6,25} \cong N_{32}$, $\mathcal{N}_{6,26} \cong N_{103}, \mathcal{N}_{6,27} \cong N_{108}, \mathcal{N}_{6,28} \cong N_{40}^{\mathrm{op}}, \mathcal{N}_{6,29} \cong N_{107}, \mathcal{N}_{6,30} \cong N_{102}^{\mathrm{op}}, \mathcal{N}_{6,31} \cong N_{109}, \mathcal{N}_{6,32} \cong N_{86}^{\mathrm{op}}$, $\mathcal{N}_{6,33} \cong N_{104}, \mathcal{N}_{6,34} \cong N_{36}, \mathcal{N}_{6,35} \cong N_{39}^{\mathrm{op}}, \mathcal{N}_{6,36} \cong N_{106}, \mathcal{N}_{6,37} \cong N_{100}^{\mathrm{op}}, \quad \mathcal{N}_{6,38} \cong N_{110}^{\mathrm{op}}, \mathcal{N}_{6,39} \cong N_{89}^{\mathrm{op}}$, $\mathcal{N}_{6,40} \cong N_{37}^{\mathrm{op}}, \mathcal{N}_{6,41} \cong N_{75}, \mathcal{N}_{6,42} \cong N_{98}^{\mathrm{op}}, \mathcal{N}_{6,43} \cong N_{111}^{\mathrm{op}}, \mathcal{N}_{6,44} \cong N_{91}^{\mathrm{op}}, \mathcal{N}_{6,45} \cong N_{76}^{\mathrm{op}}, \mathcal{N}_{6,46} \cong N_{97}$, and $\mathcal{N}_{6,47} \cong N_{93}^{\mathrm{op}}$.

Step II. We now describe (to within a strong isomorphism) all couples $(Y, X)$ of proper lower subsets in the critical Nazarova sets $\mathcal{N}_{2}-\mathcal{N}_{6}$ such that $X \subseteq Y$ and $X<S \backslash Y$ (for $\mathcal{N}_{1}$, these couples are absent):

for $\mathcal{N}_{2} \quad B_{2,1}=\left(A_{2,10},\{4\}\right)$;

for $\mathcal{N}_{3} \quad B_{3,1}=\left(A_{3,18},\{5\}\right), B_{3,2}=\left(A_{3,22},\{5\}\right), B_{3,3}=\left(A_{3,22},\{5,6\}\right)$, and $B_{3,4}=\left(A_{3,23},\{3\}\right)$;

for $\mathcal{N}_{4} \quad B_{4,1}=\left(A_{4,25},\{5\}\right), \quad B_{4,2}=\left(A_{4,32},\{5\}\right), \quad B_{4,3}=\left(A_{4,32},\{5,6\}\right), \quad B_{4,4}=\left(A_{4,34},\{2\}\right), \quad B_{4,5}=$ $\left(A_{4,37},\{5\}\right), B_{4,6}=\left(A_{4,37},\{5,6\}\right), B_{4,7}=\left(A_{4,37},\{5,6,7\}\right), B_{4,8}=\left(A_{4,38},\{2\}\right)$, and $B_{4,9}=$ $\left(A_{4,38},\{2,3\}\right)$;

for $\mathcal{N}_{5} \quad B_{5,1}=\left(A_{5,16},\{4\}\right), \quad B_{5,2}=\left(A_{5,22},\{4\}\right), \quad B_{5,3}=\left(A_{5,22},\{4,5\}\right), \quad B_{5,4}=\left(A_{5,28},\{4\}\right)$, $B_{5,5}=\left(A_{5,28},\{4,5\}\right), \quad B_{5,6}=\left(A_{5,28},\{4,5,6\}\right), \quad B_{5,7}=\left(A_{5,34},\{4\}\right), \quad B_{5,8}=$ $\left(A_{5,34},\{4,5\}\right), \quad B_{5,9}=\left(A_{5,34},\{4,5,6\}\right), \quad B_{5,10}=\left(A_{5,34},\{4,5,6,7\}\right), \quad B_{5,11}=$ $\left(A_{5,39},\{4\}\right), \quad B_{5,12}=\left(A_{5,39},\{4,5\}\right), \quad B_{5,13}=\left(A_{5,39},\{4,5,6\}\right), \quad B_{5,14}=\left(A_{5,39},\{4\right.$, $5,6,7\}), B_{5,14}=\left(A_{5,39},\{4,5,6,7,8\}\right)$, and $B_{5,16}=\left(A_{5,40},\{2\}\right)$;

for $\mathcal{N}_{6} \quad B_{6,1}=\left(A_{6,32},\{1\}\right), \quad B_{6,2}=\left(A_{6,39},\{1\}\right), \quad B_{6,3}=\left(A_{6,39},\{1,2\}\right), \quad B_{6,4}=\left(A_{6,41},\{6\}\right), \quad B_{6,5}=$ $\left(A_{6,44},\{1\}\right), \quad B_{6,6}=\left(A_{6,44},\{1,2\}\right), \quad B_{6,7}=\left(A_{6,44},\{1,2,3\}\right), \quad B_{6,8}=\left(A_{6,45},\{6\}\right), \quad B_{6,9}=$ 
$\left(A_{6,45},\{8\}\right), \quad B_{6,10}=\left(A_{6,45},\{6,8\}\right), B_{6,11}=\left(A_{6,46},\{6\}\right), B_{6,12}=\left(A_{6,47},\{1\}\right), B_{6,13}=\left(A_{6,47}\right.$, $\{1,2\}), B_{6,14}=\left(A_{6,47},\{1,2,3\}\right)$, and $B_{6,15}=\left(A_{6,47},\{1,2,3,4\}\right)$.

By $N_{i, j}^{\prime}$ we denote the poset $\left(S_{Y}^{\uparrow}\right)_{X}^{\uparrow}$ for $S=\mathcal{N}_{i}$ and $(Y, X)=B_{i, j}$. Then it is easy to see that $N_{2,1}^{\prime} \cong N_{42}$, $N_{3,1}^{\prime} \cong N_{3}^{\mathrm{op}}, \quad N_{3,2}^{\prime} \cong N_{48}, \quad N_{3,3}^{\prime} \cong N_{3}, N_{3,4}^{\prime} \cong N_{5}, \quad N_{4,1}^{\prime} \cong N_{9}^{\mathrm{op}}, N_{4,2}^{\prime} \cong N_{57}^{\mathrm{op}}, N_{4,3}^{\prime} \cong N_{10}, \quad N_{4,4}^{\prime} \cong N_{12}^{\mathrm{op}}$, $N_{4,5}^{\prime} \cong N_{61}, N_{4,6}^{\prime} \cong N_{57}, N_{4,7}^{\prime} \cong N_{9}, N_{4,8}^{\prime} \cong N_{59}, N_{4,9}^{\prime} \cong N_{12}, \quad N_{5,1}^{\prime} \cong N_{20}^{\mathrm{op}}, N_{5,2}^{\prime} \cong N_{72}^{\mathrm{op}}, N_{5,3}^{\prime} \cong$ $N_{21}^{\mathrm{op}}, \quad N_{5,4}^{\prime} \cong N_{78}^{\mathrm{op}}, \quad N_{5,5}^{\prime} \cong N_{73}^{\mathrm{op}}, \quad N_{5,6}^{\prime} \cong N_{22}, \quad N_{5,7}^{\prime} \cong N_{81}^{\mathrm{op}}, \quad N_{5,8}^{\prime} \cong N_{79}, N_{5,9}^{\prime} \cong N_{73}, \quad N_{5,10}^{\prime} \cong N_{21}$, $N_{5,11}^{\prime} \cong N_{83}, \quad N_{5,12}^{\prime} \cong N_{81}, N_{5,13}^{\prime} \cong N_{78}, N_{5,14}^{\prime} \cong N_{72}, N_{5,15}^{\prime} \cong N_{20}, N_{5,16}^{\prime} \cong N_{24}, N_{6,1}^{\prime} \cong N_{33}^{\mathrm{op}}$, $N_{6,2}^{\prime} \cong N_{87}^{\mathrm{op}}, \quad N_{6,3}^{\prime} \cong N_{34}^{\mathrm{op}}, N_{6,4}^{\prime} \cong N_{35}, N_{6,5}^{\prime} \cong N_{90}^{\mathrm{op}}, N_{6,6}^{\prime} \cong N_{88}, N_{6,7}^{\prime} \cong N_{34}, N_{6,8}^{\prime} \cong N_{105}, N_{6,9}^{\prime} \cong N_{38}^{\mathrm{op}}$, $N_{6,10}^{\prime} \cong N_{35}^{\mathrm{op}}, N_{6,11}^{\prime} \cong N_{38}, \quad N_{6,12}^{\prime} \cong N_{92}, N_{6,13}^{\prime} \cong N_{90}, N_{6,14}^{\prime} \cong N_{87}$, and $N_{6,15}^{\prime} \cong N_{33}$.

Step III. It is easy to see that each poset $N_{i}$ and $N_{i}^{\text {op }}$, where $i=1,2, \ldots, 115$, appears only once in Steps I and II (moreover, if $N_{i}^{\mathrm{op}} \cong N_{i}$, then $N_{i}$ is present but $N_{i}^{\mathrm{op}}$ is absent).

Theorem 1 is thus proved.

\section{Tables}

Table 1. P-Critical Posets

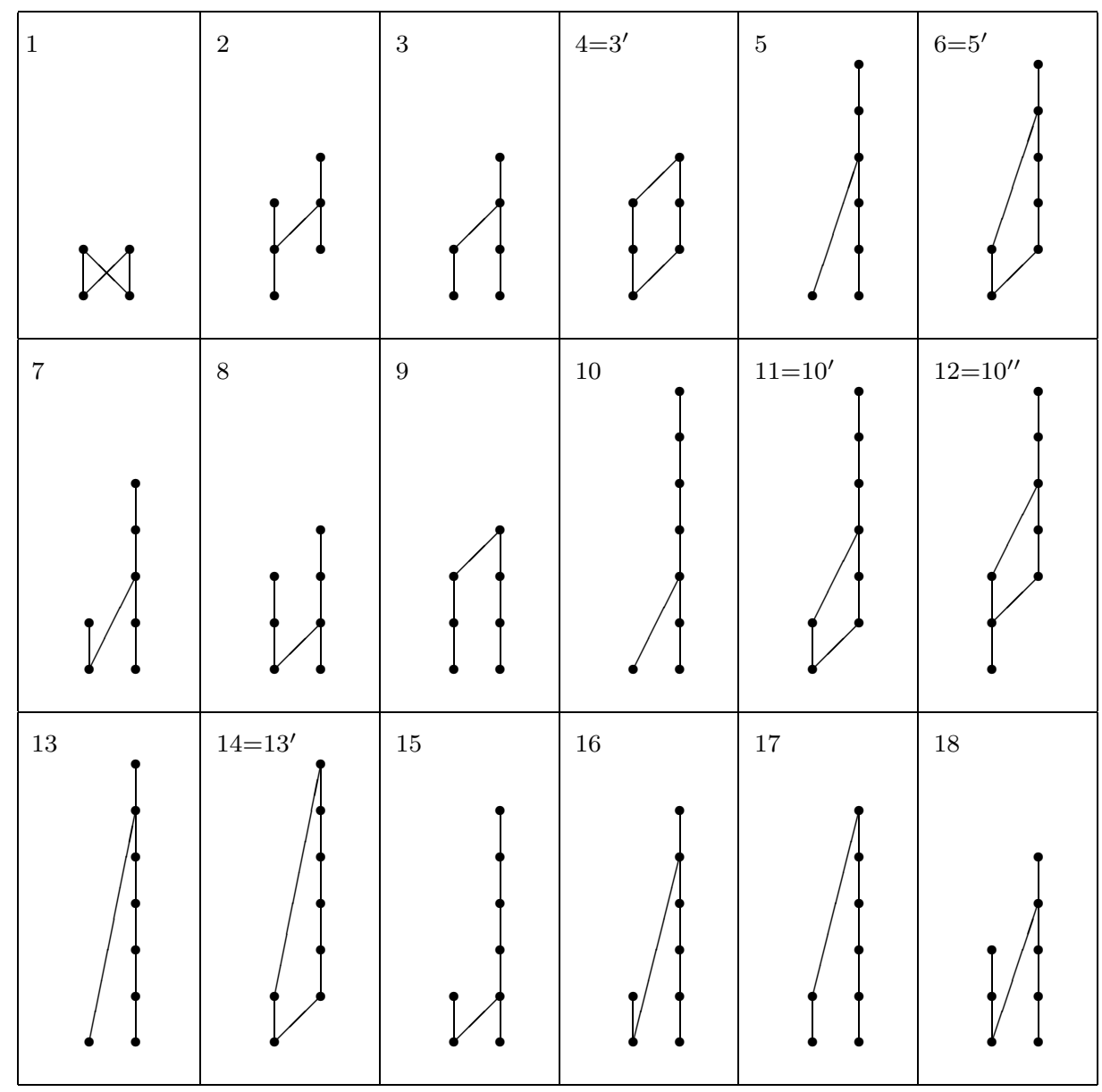


Table 1

(continued)

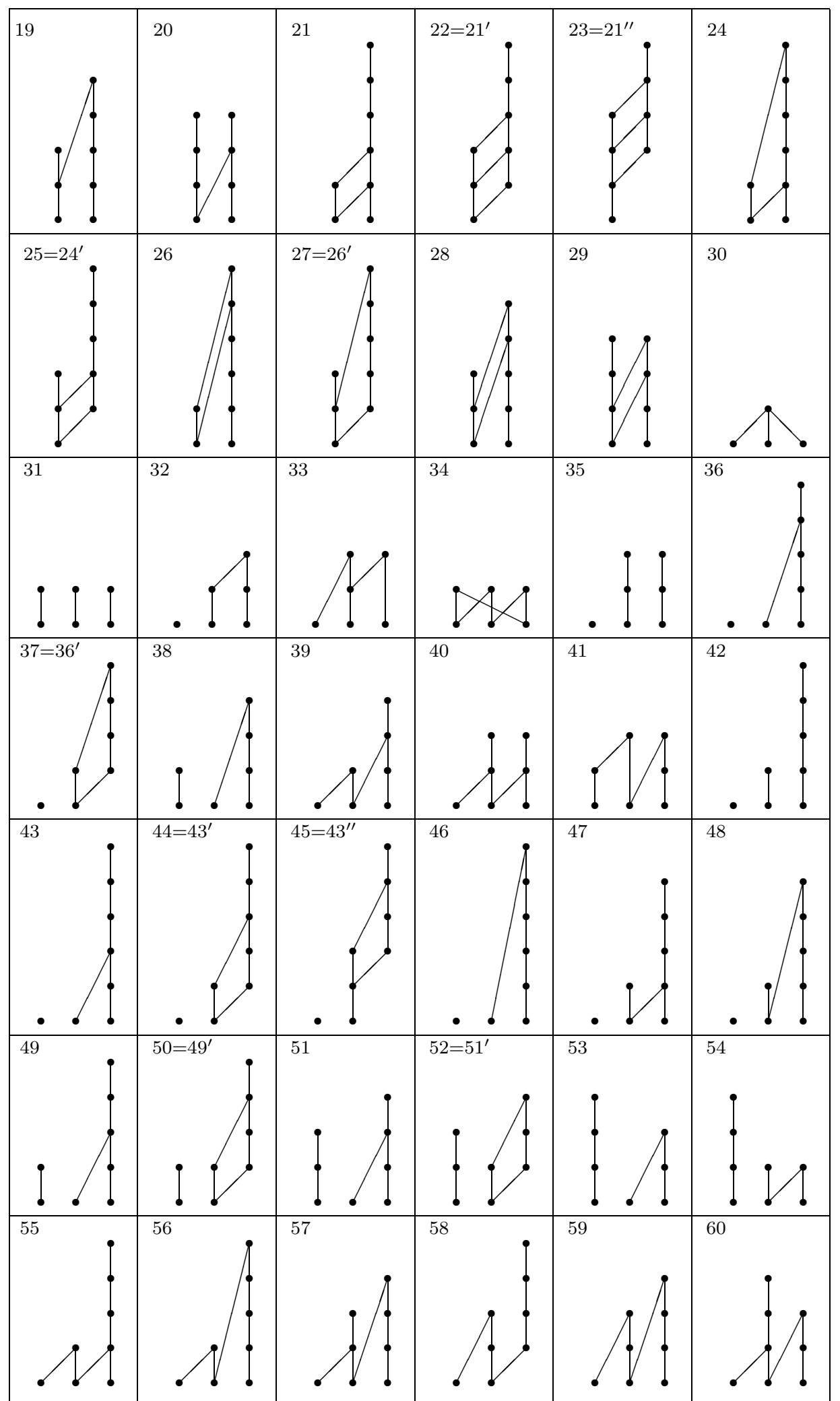


742

V. M. BONDARENKO AND M. V. STEPOCHKINA

Table 1

(continued)

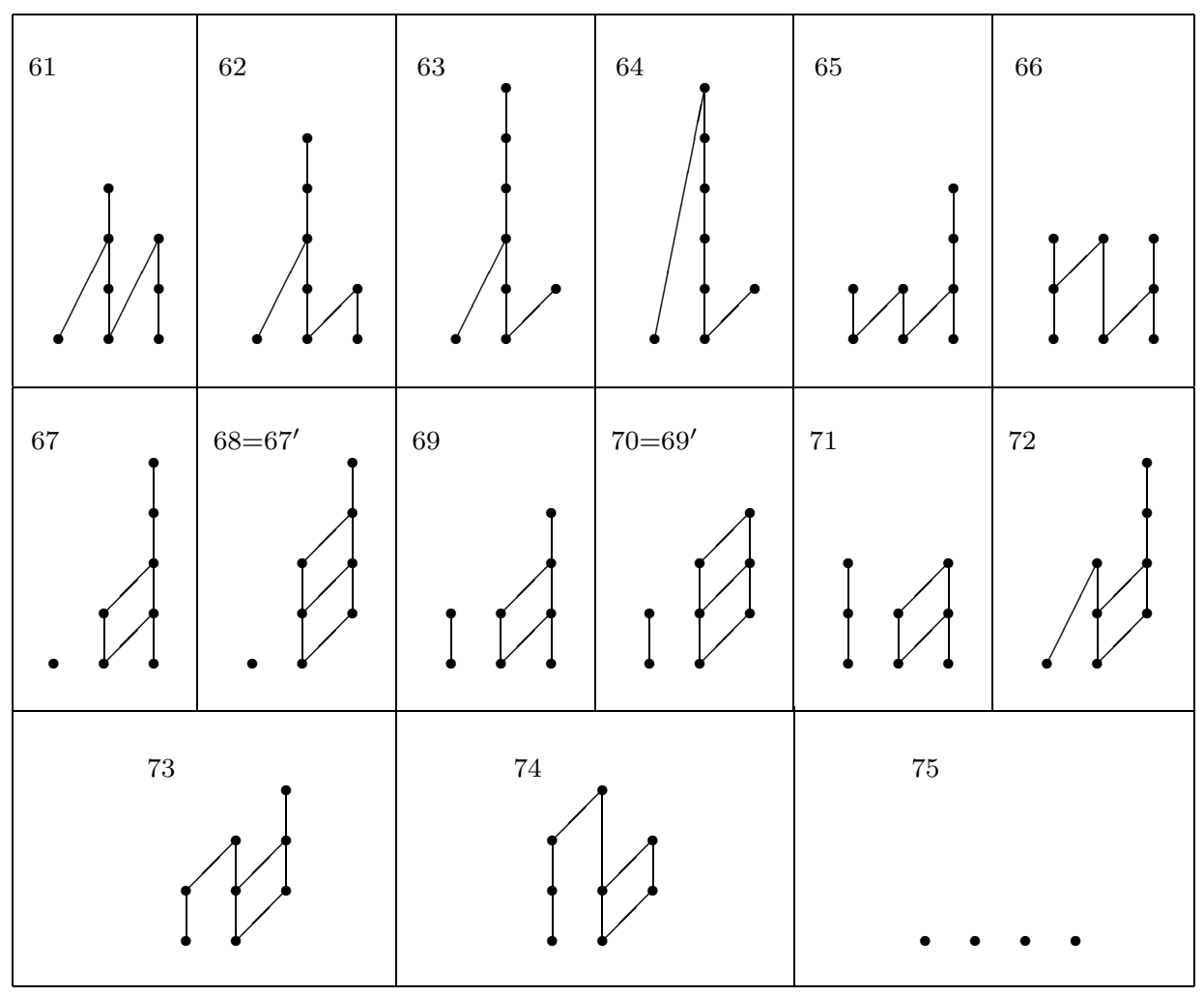

Table 2. NP-Critical Posets

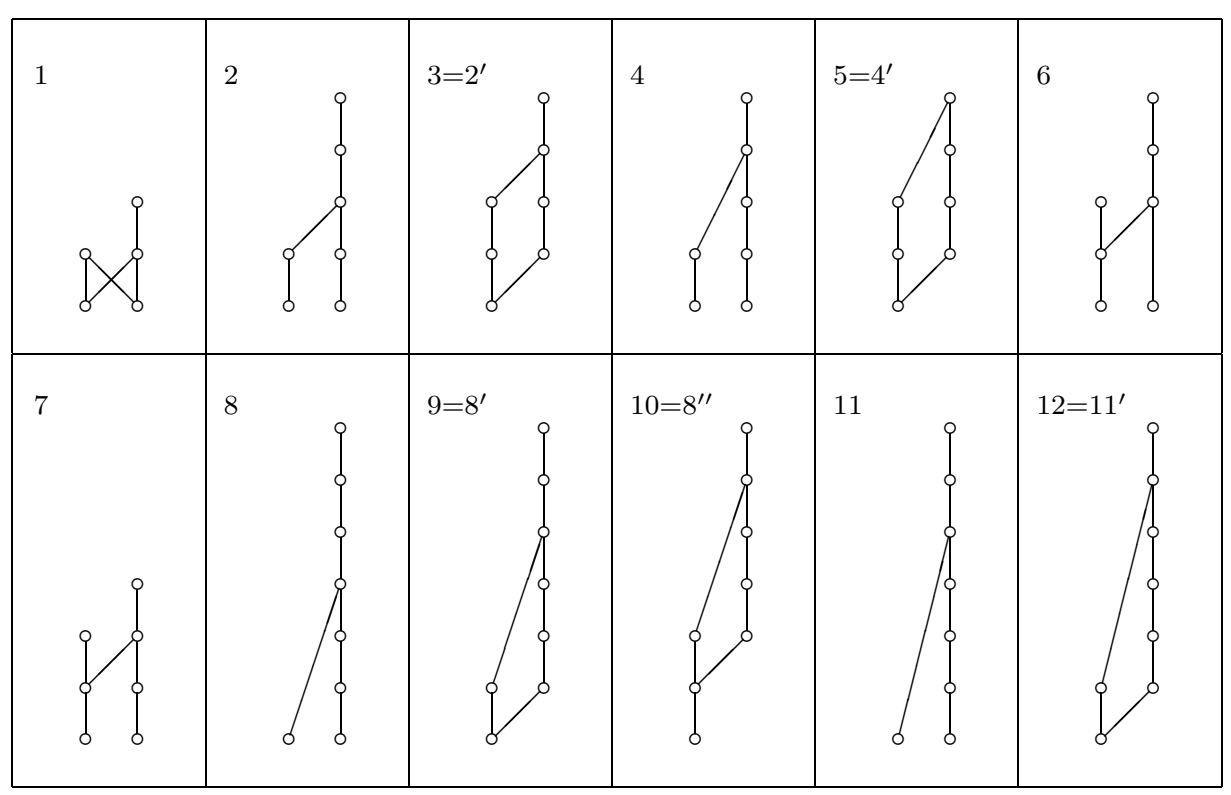


Table 2

(continued)

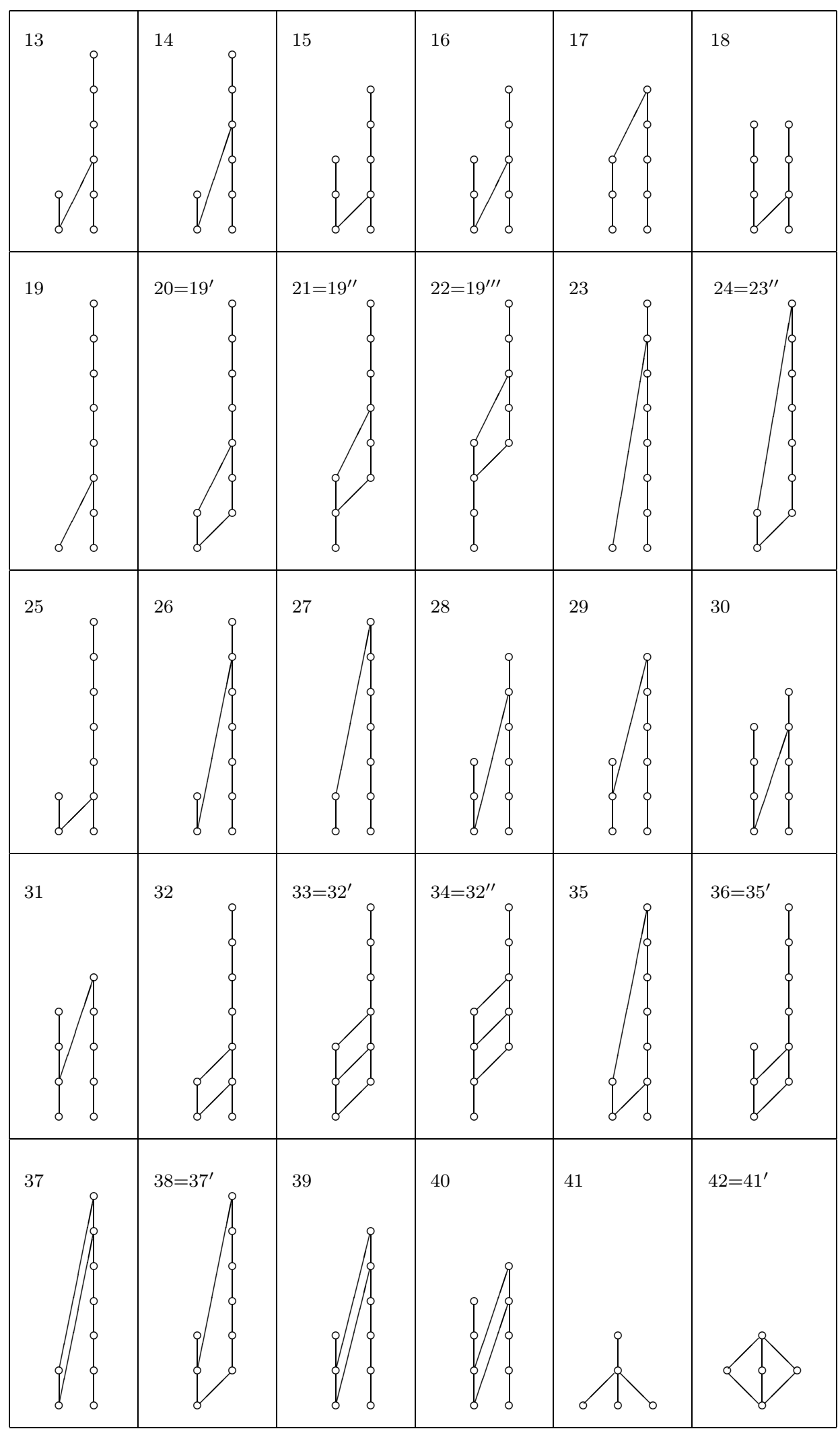


Table 2

(continued)

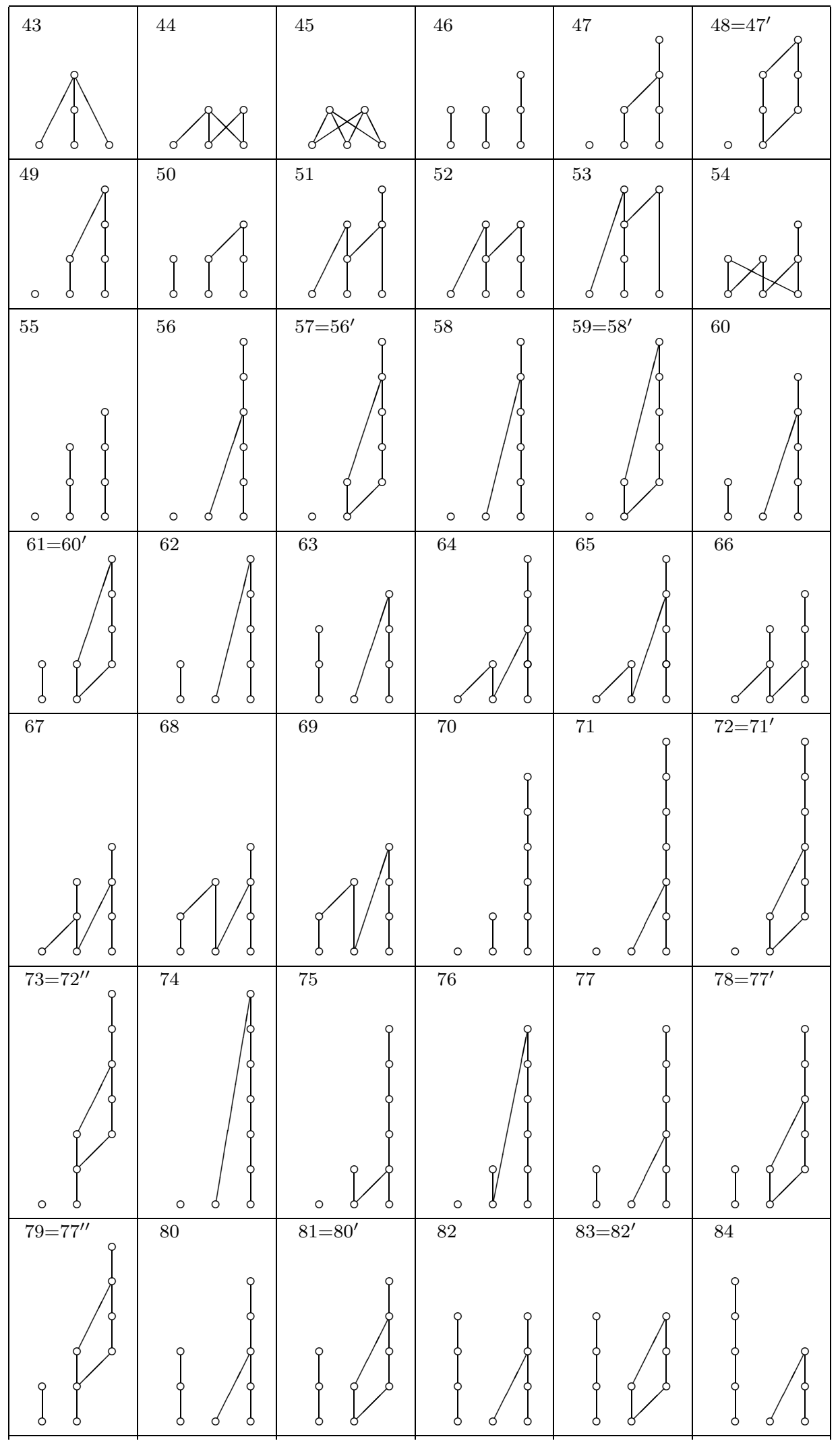


Table 2

(continued)

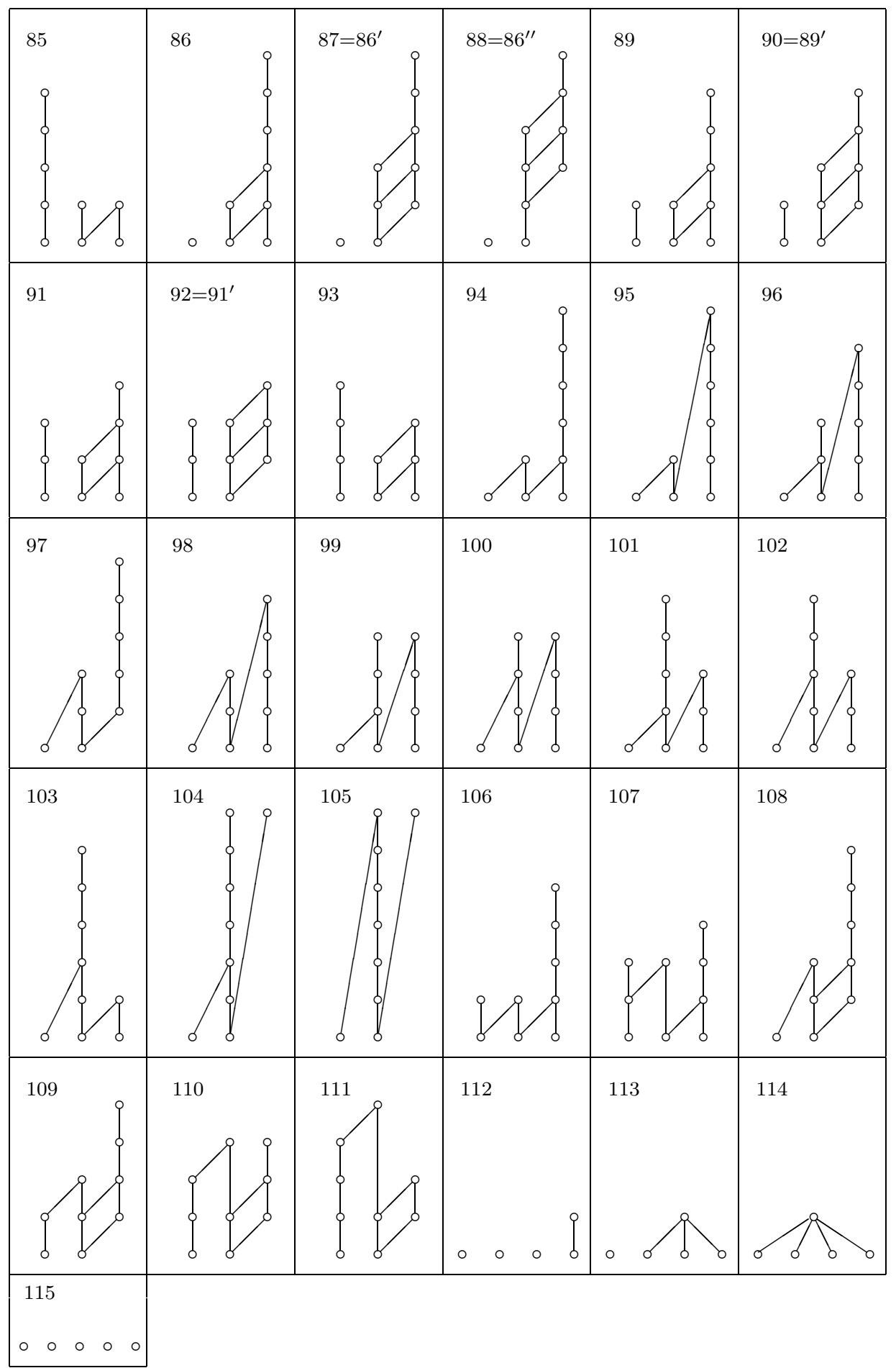

\section{REFERENCES}

1. P. Gabriel, “Unzerlegbare Darstellungen,” Manuscr. Math., 6, 71-103, 309 (1972). 
2. S. Brenner, "Quivers with commutativity conditions and some phenomenology of forms," in: Proc. of the Internat. Conf. on Representations of Algebras, Carleton University, Ottawa (1974), Paper No. 5.

3. Yu. A. Drozd, "Coxeter transformations and representations of posets," Funkts. Anal. Prilozhen., 8, 34-42 (1974).

4. M. M. Kleiner and A. V. Roiter, "Representations of differential graded categories," in: Matrix Problems [in Russian], Institute of Mathematics, Academy of Sciences of the Ukrainian SSR (1977), pp. 5-70.

5. L. A. Nazarova and A. V. Roiter, "Representations of posets," Zap. Nauch. Semin. LOMI, 28, 5-31 (1972).

6. V. M. Bondarenko and A. M. Polishchuk, "On the criterion of positive definiteness for one class of infinite quadratic forms," Nelin. Kolyv., 6, No. 1, 3-14 (2003).

7. V. M. Bondarenko, "On (min, max)-equivalence of posets and applications to the Tits forms," Visn. Kyiv Univ., Ser. Fiz. Mat., No. 1, 24-25 (2005).

8. V. M. Bondarenko and M. V. Styopochkina, "On posets of width two with positive Tits form," Algebra Discrete Math., No. 2, 11-22 (2005).

9. V. M. Bondarenko and M. V. Stepochkina, "Posets of the injective-finite type," Nauk. Visn. Uzhhorod Univ., Ser. Mat. Inform., Issue 9, 15-25 (2005).

10. V. M. Bondarenko and M. V. Stepochkina, “(Min, max)-equivalence of posets and the quadratic Tits form,” in: Collection of Works "Problems of Analysis and Algebra," Institute of Mathematics, Ukrainian National Academy of Sciences, 2, No. 3 (2005), pp. 18-58.

11. V. M. Bondarenko and M. V. Styopochkina, "On finite posets of inj-finite type and their Tits forms," Algebra Discrete Math., No. 2, 17-21 (2006).

12. V. M. Bondarenko and M. V. St'opochkina, "On the serial posets with positive-definite quadratic Tits form," Nelin. Kolyv., 9, No. 3, 320-325 (2006).

13. V. M. Bondarenko and M. V. St'opochkina, "On the form of posets with positive-definite Tits form," Visn. Kyiv Univ., Issue 3, 11-14 (2006).

14. V. M. Bondarenko and M. V. St'opochkina, "On the relationship between the inj-finiteness of the type and positive-definiteness of the quadratic Tits form for finite posets," Nauk. Visn. Uzhhorod Univ., Issue 12, $20-27$ ( 2006).

15. V. M. Bondarenko and M. V. Stepochkina, "(Min, max)-equivalence of posets and nonnegative Tits forms," Ukr. Mat. Zh., 60, No. 9, 1157-1167 (2008). 